\title{
Atenção básica e saúde pública: por que e para que compreender o papel do agente na sua formação e preparação dos primeiros atendimentos de saúde
}

\author{
Wener Maria Santos Flor de Lima
}

\section{RESUMO}

Motivação: Este trabalho aborda a importância do conhecimento sobre saúde pública e atenção básica, que hoje, nos termos de formação dos profissionais desta área, apresenta um quadro bem diversificado com orientações que favorecem ações para indivíduos que necessitam de cuidados em atenções primárias que abrangem a saúde dos indivíduos. Leva-se em conta a identificação de métodos utilizados para avaliação de incidentes na atenção primária a saúde, os tipos, os fatores contribuintes e as soluções para tornar a atenção inicial a saúde mais segura. Tendo como objetivo principal trazer informações a cerca da atenção básica e conhecer a importância deste debate para a formação profissional do agente de saúde.

Método: Metodologicamente o estudo foi realizado a partir da pesquisa bibliográfica, descritiva sistêmica referenciada, onde os autores debatem sobre o tema.

Resultados: Os resultados esbaram em teorias neste campo emergencial que é a saúde primária e a responsabilidade do profissional contracenando com críticas e reflexões pertinentes sobre $o$ assunto.

Conclusões: Foi possível analisar a importância, efetividade e responsabilidade que o Agente de saúde tem nos cuidados de atenção básica, tornando-se indispensável seu papel como agente transformador no que tange a saúde e bem estar dos indivíduos.

Palavras-chave: Saúde Pública; Doenças; Atenção Primária; Agente de Saúde.
Revista da Rede APS 2019

Publicada em: 17/12/2019

DOI:10.14295/aps.v1i3.44

Autores Wener Maria

Santos Flor de Lima

(Universidade da Amazônia, Manaus, Amazonas, Brasil);

Correspondência para: Autores Wener Maria Santos Flor de Lima wener.tec@hotmail.com 


\section{INTRODUÇÃO}

Em primeiro plano, o campo da saúde se constitui em um fiel medidor das condições de vida de um povo, partindo desta premissa, percebe-se o quanto é importante conhecermos a fundo sobre a atenção primária e algumas reflexões em prol dos primeiros atendimentos e cuidados sobre a saúde no campo da atenção básica. A preocupação com as informações passadas para os pacientes levou a $\mathrm{OMS}^{1}$ a criar vários programas com um objetivo claro de desenvolver políticas mundiais para melhorar os cuidados aos pacientes nos serviços de saúde pública.

Entre as iniciativas desses programas, que se deu a partir de 2004, destaca-se a tentativa de conceituar as questões envolvidas com a segurança do paciente. Foi desenvolvida a Classificação Internacional de Segurança do Paciente, na qual incidente é definido como todo evento ou circunstância que poderia ter resultado, ou resultou, em dano desnecessário ao paciente. (BRASIL, 2014).

Por isso, em segunda análise, considera-se que para se ter uma qualidade de vida com prevenções em torno da saúde, deve-se compreender que tal "qualidade" está diretamente formada pelos interesses de cada indivíduo junto à sua formação ética, cívica e profissional e principalmente do conhecimento de cada sujeito.

Neste caso, a relevância deste estudo envolto dos cuidados básicos a saúde e seu oferecimento à sociedade em relação à "qualidade de vida" necessária e urgente, é uma referência constante do discurso político, este também presente, ainda que com entendimentos distintos, nos desejos e aspirações dos indivíduos de cada setor da saúde pública ou no meio social ao qual pertence.

\footnotetext{
${ }^{1}$ Organização Mundial de Saúde
}

Nesse sentido, a atenção primária é uma ferramenta de auxílio no processo de desenvolvimento educacional, social e de saúde do ser humano, assim, é necessário à reflexão e discussão sobre como o conceito da atenção básica está inserido no meio hospitalar em possíveis intervenções de discussões clínicas.

Portanto, este estudo justifica-se a partir da proposição através de um dos seus objetivos que é a avaliação desta atenção primária que pode ser um fator de coesão com a imagem do agente de saúde onde se procura levar em conta a diversidade dos indivíduos e dos grupos humanos, evitando tornar-se um fator de exclusão social ou de manipulação territorial essa não preocupação com a saúde.

Desse modo é um assunto que está ligado a um modelo social industrial e capitalista que cresce continuamente enraigado na supremacia do homem sobre a natureza e suas possibilidades, sob a luz de discussões e debates sobre o objetivo geral centrado na saúde ao passo de palavras como resultados de muito estudos sobre esse fenômeno e nas inovações do processo histórico que faz da "atenção" um processo de ordem obrigatório de sobrevivência, visando sobre a ordem de: "fortalecer a participação e o controle social sobre todas as instâncias e dos envolvidos nesta área".

Voltado para o interesse maior desta discussão de base sistêmica referencial e documental.

\section{Diagnóstico dA Atenção BÁsıCA EM}

\section{SAÚDE: BREVES COMENTÁRIOS}

Diante da atual conjuntura social, nas discursões acerca da saúde pública, percebemse problemas no modelo assistencial - forma como se dá o atendimento em saúde - é um modelo fragmentado, especializado, compartimentalizado, não atendendo as reais necessidades das pessoas e sem considerar de forma integral, restabelecendo a saúde de quem precisa. É como se trabalhássemos em 
vão, pois não se tem saúde e nem meios estruturais para o paciente receber $\mathrm{ABS}^{2}$. É necessário que o discurso seja realizado aqui, visando uma reflexão voltada para um debate possível, passando a dividir a responsabilidades com todos os envolvidos.

Neste caso, tem que ser um trabalho coletivo e não individualizado, interagindo com o paciente e a comunidade, através de uma abordagem integral. Isto faz uma diferença determinante. (ABBAS, 2005, p. 34) nos alerta de que: "antes se esperava as pessoas adoecerem para se atuar sobre indivíduos em sofrimento"; agora se atua com cidadãos saudáveis com o objetivo de manter a qualidade de vida e buscá-la, se ainda não foi alcançada.

\begin{abstract}
Atenção Básica caracteriza-se por um conjunto de ações de saúde, no âmbito individual e coletivo, que abrangem a promoção e a proteção da saúde, a prevenção de agravos, o diagnóstico, o tratamento, a reabilitação e a manutenção da saúde. É desenvolvida por meio do exercício de práticas gerenciais e sanitárias democráticas e participativas, sob forma de trabalho em equipe, dirigidas a populações de territórios bem delimitados, pelas quais assume a responsabilidade sanitária, considerando a dinamicidade existente no território em que vivem essas populações. (BRASIL, 2006, p. 1).
\end{abstract}

Contudo, essa coletividade expressa no exceto acima, utiliza-se de tecnologias e de elevada complexidade e baixa densidade, que devem resolver os problemas de saúde de maior frequência e relevância em seu território. É o contato preferencial dos usuários com os sistemas de saúde. Orienta-se pelos princípios da universalidade, da acessibilidade e da coordenação do cuidado, do vínculo e continuidade, da integralidade, da responsabilização, da humanização, da equidade e da participação social.

\footnotetext{
${ }^{2}$ Atenção Básica a Saúde
}

\footnotetext{
${ }^{3}$ Sistema Único de Saúde
}

Assim, cada serviço tem suas características próprias, sua função bem definida e densidades tecnológicas distintas na rede. Essa organização possibilita a comunicação de forma horizontal, não autoritária. (MENDES, 2008, p. 35), e continua conceituando as redes,

A atenção básica em saúde na Rede, não tem, por si só, capacidade de responder a toda gama de serviços, mas tenta resolver alguns quisitos que permeiam esta problemática, com ações envolvendo a construção de relações neste meio, tais como níveis de associações; cooperações; colaborações; conhecimentos e reconhecimentos. Em ações de compartilhamentos e projetos com atividades e recursos para prestar ajuda esporádica, conhecendo o que o outro é e faz, com total reconhecimento ao existir do outro, total cumplicidade. Assim, buscando valores de confiança, solidariedade, reciprocidade, interesse e aceitação. (MENDES, 2008).

Em linhas gerais, uma proposta interessante para a total democratização da atenção básica em relação aos primeiros atendimentos a saúde e sobre a formação das redes são muito importantes e podem ser instrumentos de grande valia para a operacionalização das redes de saúde no SUS 3 .

Assim, outra preocupação neste tópico são os fatores contribuintes para a não incidência desta rede nos rol da saúde pública. Ocorre: falhas na comunicação interpessoal e com os pacientes; despreparo de agentes de saúdes; falhas na gestão, tais como: falta de insumos medico-cirúrgicos e de medicamentos, profissionais pressionados para serem mais produtivos em menos tempo, falhas em prontuários, falhas na recepção dos pacientes, planta física da unidade de saúde inadequada, descarte inadequado de resíduos da unidade de saúde, tarefas excessivas e falhas no cuidado. Um risco que precisa de reparos emergenciais. 
ISSN 2596-3317 - DOI 10.14295/aps.v1i3.44

Lima, W. M. S. F.

\section{Segurança do Paciente na Atenção} INICIAL À SAÚDE

Neste estudo sobre a segurança do paciente em atenção primária à saúde, percebe-se que vem crescendo sua importância nas principais organizações internacionais de saúde. "No campo da atenção primária à saúde há espaço para estudos sobre a segurança do paciente, visto que, a grande maioria dos cuidados em saúde acontece na atenção primária à saúde". (MAKEHAM, 2008, p. 11).

Indubitavelmente, a segurança do paciente, partindo da atenção básica, deverá estar focalizada na identificação da direção mais adequada de utilização dos serviços, com o objetivo de completar a atenção brindada na atenção básica e propiciando o menor tempo possível nesse trajeto e na oportunidade necessária para evitar o agravamento de problemas de saúde.

“A flexibilização do processo de trabalho, balizado pelas determinantes sociais incorporadas na interação, possibilitará que a concepção sistêmica adotada nesse referencial de avaliação seja a de um sistema aberto, que levará em conta o poder de utilização do usuário para ampliar a acessibilidade à $\mathrm{ABS}^{\prime \prime}$. (NOGUEIRA, 2005, p. 45).

Neste aspecto, a tomada de decisão, ou seja, a mobilização de recursos para os meios de proporcionar a segurança ao paciente, será destinada a realizar os ajustes em pleno processo, de forma dinâmica e oportuna, não precisando necessariamente aguardar resultados dos exames, seria uma forma de relacionar a objetividade com o caráter introspectivo da realização do processo inicial.

\section{Atenção Básica: SAÚde da CRIANÇA}

A saúde da criança é a base prioritária de qualquer entidade ou instituição médica. Neste sentido, no tocante à saúde da criança, em 2015, o Ministério da Saúde instituiu o
(PNAISC) $^{4}$ com a Portaria no 1.1303, a qual sintetiza de maneira clara e objetiva os eixos de ações que compõem a atenção integral à saúde da criança. $O$ documento aponta estratégias e dispositivos para a articulação das ações e dos serviços de saúde, a fim de facilitar sua implementação pelas gestões estadual e municipal e pelos profissionais de saúde.

Nesses termos, efetiva-se a capacidade que o controle social possui para intervir na política de saúde em relação à criança, apresentando estratégias de fortalecimento das políticas de democratização do SUS e, consequentemente, de resistência à privatização da saúde.

O referencial teórico deste estudo, um serviço de atenção básica para ser considerado provedor, isto é, orientado à Atenção Primária à Saúde destinados as crianças, devem apresentar os atributos ordenadores. Ressaltase que só haverá atenção de qualidade as crianças de qualidade quando seus sete atributos estiverem sendo operacionalizados em sua totalidade. (STARFIELD, 2002, P. 34).

São eles segundo Starfield (2002): primeiramente, possuir o acesso necessário para apresentar evidências consistentes para a associação entre maior grau de orientação à atenção básica inicial, segundo propiciar longitudinalidade com o aumento da efetividade dos sistemas de saúde. Em terceiro, integralidade emconsonância e mais instabilidade dessa realidade e no âmbito da saúde da criança. Em quarto uma coordenação segura com os serviços orientados a atenção sendo capazes de reduzir as internações hospitalares por condições sensíveis à atenção primária.

Ainda segundo o autor, em quinto, orientação, contribuindo para a redução do índice de internações na área da saúde infantil. Em sexto, a família como importante parceria, fato que ainda não é observado no contexto da saúde. $E$ por último a comunidade, voltado para união e

\footnotetext{
${ }^{4}$ Política Nacional de Atenção Integral à Saúde da Criança
} 
informações, cujas principais causas de não atenção as internações pediátricas são devidas a condições sensíveis à atenção básica a exemplo dos problemas respiratórios e outros similares.

Doravante, como consequência de todos os itens mencionados acima, diversos problemas aparecem neste meio, dessa forma de atender às crianças, tem-se a fragilidade no estabelecimento do vínculo entre os envolvidos no cuidado, com isso a responsabilidade maior é do agente de saúde.

\section{Políticas Públicas de Saúde em ATENÇÃo BÁSICA}

Historicamente as políticas públicas, em especial no Brasil, caracterizam-se como subordinadas aos interesses econômicos e políticos, sendo implementadas através de práticas assistencialistas e clientelistas. Essas conservam em sua concretização o caráter fragmentário, setorial e emergencial que são reforçados gradativamente a partir da ofensiva neoliberal, que preconiza a retração do Estado na orbita da responsabilidade social, ou ainda a redução do investimento público nos serviços sociais.

Nessa perspectiva, busca-se com este tópico pontuar que a gestão das políticas públicas que devem possuir um caráter de respeito à diversidade, controle e participação social, e ênfase nas necessidades da população carente, principalmente no que tange a atenção básica.

O processo histórico acerca da Política de Saúde é longo. Partiremos da década de 1980, com a construção do Projeto de Reforma Sanitária podemos dizer que nos anos 80 , a sociedade brasileira ao mesmo tempo em que vivenciou um processo de democratização política superando $o$ regime ditatorial instaurado em 64, experimentou uma profunda e prolongada crise econômica que persiste até os dias atuais.
Então, a saúde deixou de ser interesse apenas dos técnicos para assumir uma dimensão política, estando estreitamente vinculada à democracia.

As principais propostas debatidas por esses sujeitos coletivos sobre as políticas públicas foram à universalização do acesso as ações de saúde; a concepção de saúde como direito social e dever do Estado; a reestruturação do setor através da estratégia do Sistema Unificado de Saúde visando um profundo reordenamento setorial com um novo olhar sobre a saúde individual e coletiva; a descentralização do processo decisório para as esferas estadual e municipal, o financiamento efetivo e a democratização do poder local através de novos mecanismos de gestão - os Conselhos de Saúde. "Afinal, os direitos como em todo são indispensáveis para que se possa pensar nessa forma democrática e justa de vida. No mínimo por isso deveriam ser sempre plenamente valorizados e defendidos". (NOGUEIRA 2005, p. 7).

Percebeu-se, então, a emergência e a necessidade de uma mudança na conjuntura política da saúde no Brasil e a reorientação das práticas de saúde. Para tanto, o SUS em seu arcabouço de leis, normas, resoluções e diretrizes é um marco definitivo na garantia do direito a saúde do cidadão brasileiro, por determinar um caráter universal às ações e aos serviços no país. (BRASIL, 2006, p.5).

O princípio da universalidade corresponde ao direito da saúde para todos e cabe ao Estado garantir esse direito aos brasileiros; o segundo é um princípio de justiça social e se trata do aspecto em que todos devem ter igual oportunidade em usar o sistema de saúde no Brasil; e o terceiro princípio se refere a um atendimento integral que contemple as necessidades da população incluindo tanto os meios curativos quanto os preventivos, como também uma atenção à saúde individual e coletiva, mais o que ocorre é outra realidade. 
ISSN 2596-3317 - DOI 10.14295/aps.v1i3.44

Lima, W. M. S. F.

\section{Políticas Publicas de saúde: Novos}

\section{Rumos}

A partir do pressuposto de que o SUS foi criado para oferecer atendimento igualitário e cuidar e promover a saúde de toda a população. 0 Sistema constitui um projeto social único que se materializa por meio de ações de promoção, prevenção e assistência à saúde dos brasileiros. (BRASIL, 2010).

Em linhas gerais as conquistas do Sistema Único de Saúde (SUS) nos desafiam a desenvolver propostas de intervenção que favoreçam o seu aperfeiçoamento. Diante dos obstáculos de ordem ética, política, financeira e a organização do sistema de saúde, faz-se necessário o debate sobre a humanização, questionando o modelo tecnoassistencial e a qualidade da atenção.

Os novos rumos da atenção básica a saúde juntamente com políticas públicas favoráveis, está fundamentada segundo (BARDIN, 2008) em três princípios estruturantes: a transversalidade, indicando a ampliação do grau de comunicação entre os sujeitos e serviços, visando a transformações nos territórios de poder, alterações das fronteiras dos saberes e nas relações de trabalho; a indissociabilidade entre atenção e gestão, afirmando que existe uma relação inseparável entre modos de cuidar e modos de gerir e apropriar-se do trabalho e a afirmação do protagonismo e autonomia dos sujeitos e coletivos, compreendidos como sujeitos na produção de serviços, de si próprios e do mundo, desenvolvendo atitudes de corresponsabilidade na produção de saúde.

A efetivação desses princípios estruturantes depende de uma atenção básica que seja a porta de entrada do sistema e organizadora da rede dos serviços. A humanização perpassa os processos de trabalho e os atores envolvidos na atenção básica. Por isso a importância do trabalho do agente de saúde neste contexto.

\section{O papel do Agente de Saúde em RELAÇÃO À ATENÇÃO BÁSICA}

O agente de saúde entra nesta discussão sobre os processos de trabalho na possibilidade de fortalecer o vínculo, a corresponsabilização, a participação entre gestores e trabalhadores, promovendo um ambiente saudável de trabalho, tecendo redes no sentido da participação ativa nos serviços de saúde voltados para atenção inicial em uma noção perspectiva de inovações nesta área e amor pela profissão.

Nestes termos, sendo a melhor forma de ampliar as práticas de humanização sobre este assunto, consiste na efetiva participação do agente de saúde nos processos de trabalho, com a inclusão dos sujeitos na tomada de decisões nos serviços de saúde, apontando uma gestão compartilhada como uma diretriz a ser seguida a fim de incluir novos sujeitos e multiplicar os agentes dessa política.

Assim, para compreendermos adequadamente como ocorre o processo de inserção do agente de saúde no exercício da sua profissão precisamos conhecer os objetivos a priori e suas particularidades vinculadas a um passado histórico gradual dinâmico e um processo marcante atual com transformações em vários campos de experiências profissionais e leis que regulamentam trabalhos envolvendo o setor em debate.

Ademais, é de suma importância conhecer a fundo o papel que desempenha este cidadão na construção de discursos de em prol do desenvolvimento médico no quesito "atenção básica" e suas consequências ao não cumprimento em relação ao paciente, é marcante considerar os obstáculos e alguns elementos que constitui este processo de intervenção: a imagem que o profissional faz de si e dos outros, o contexto histórico-social voltado para a realidade, às pessoas que participam do processo de interação que se dá por meio de abordagens lúdicas e proporcionais ao ambiente dos postos de 
APS em Revista

Vol. 1, n. 3, p. 181/188 | Setembro/Dezembro - 2019

ISSN 2596-3317 - DOI 10.14295/aps.v1i3.44

Lima, W. M. S. F.

saúde, os desafios desta profissão, e como ensinar métodos, regras, princípios em lugares propensos a falta de materiais necessários para $o$ atendimento eficaz.

Neste caso, a necessidade de políticas públicas que favoreçam realmente o bem estar destes indivíduos e o pleno exercício com responsabilidade e qualidade em ambientes determinados por práticas sociais que muitas vezes se perdem em teorias ou nepotismos, prevalecendo o financeiro e não o amor pelo próximo. Levar os sujeitos à construção de um mundo no qual possam viver com dignidade, consciência e compromisso, promovendo o desenvolvimento humano.

\section{CONSIDERAÇÕES FINAIS}

Este trabalho teve por objetivo básico discorrer sobre a importância do agente de saúde em relação à atenção básica ao paciente e cuidados com a saúde. Uma proposta com discurso viável e realista com autores que criticam e promovem suportes para se ter um conhecimento mais forte no tema em questão.

A postura do profissional tem que ser de agente transformador é de suma importância neste meio discursivo, pois provoca a perspectiva da sua própria competência e aprimoramento intelectual e formação de uma ordem social que visa o bom funcionamento sem exploração de classes, com qualidade e planejamento que orientam os pacientes por caminhos de participação com um aprendizado satisfatório e perspectivas grandiosas com programas e projetos sociais eficazes.

Em síntese, o estudo indicou que a humanização com afetividade visa superar o modelo biomédico-hegemônico em direção à centralidade no usuário, que é o sujeito do processo assistencial. Para tanto, as tecnologias das relações funcionam como ferramentas para a construção de vínculos e de práticas de saúde que extrapolam a visão fragmentada do cuidado.
ABBAS, A. K. Vírus da Imunodeficiência Humana

e Síndrome da Imunodeficiência Adquirida. In: Imunologia Celular e Molecular. 5.

Ed. São Paulo: Elsevier, 2005.

BRASIL. Ministério da Saúde do Brasil. Comissão Nacional de Incorporação de Tecnologias no SUS - CONITEC [Internet]. [2010]. Disponível em: http://portal.saude.gov.br/portal/saude/Gestor /area.cfm?id_area=1611. Acessado em 13/12/2019.

BRASIL. Política Nacional de Atenção Básica. Brasília: Ministério da Saúde, 2006. (Série E. Legislação de Saúde. Série Pactos pela Saúde).

BRASIL, Ministério da Saúde. Coletâneas e normas para o controle social no sistema único de saúde. Brasília: Ministério da Saúde, 2006 a.

BRASIL. Ministério da Saúde. Programa Nacional de Melhoria do Acesso e da Qualidade da Atenção Básica (PMAQ). Disponível em:

http://dab.saude.gov.br/portaldab/noticias. Php?Conteudo=28_11_Resultados_preliminares _pmaq_ab (acessado em 30/Mai/2014).

BARDIN L. Análise de conteúdo. Lisboa: Edições 70; 2008.

MAKEHAM MA, Cooper C, Kidd MR. Lessons from the TAPS study - message handling and appointment systems. Aust Fam Physical 2008; 37:438-9.

MENDES. E. V. As redes de atenção à saúde. Revista Médica de Minas Gerais, Belo Horizonte, v. 18 , n. 4 supl, p. 3-11, 2008.

NOGUEIRA, Marco Aurélio. 0 desafio de consolidar direitos no mundo globalizado. In: Revista Serviço Social e Sociedade. São Paulo; Cortez, julho, 2005.

STARFIELD B. Atenção Primária: equilíbrio entre necessidades de saúde, serviços e tecnologia. Brasília: Ministério da Saúde (MS); 2002.

\section{REFERÊNCIAS BIBLIOGRÁFICAS}




\section{ARTIGOS}

APS em Revista

Vol. 1, n. 3, p. 181/188 | Setembro/Dezembro - 2019

ISSN 2596-3317 - DOI 10.14295/aps.v1i3.44

Lima, W. M. S. F.

\section{ABSTRACT}

Motivation: This paper addresses the importance of knowledge about public health and primary care, which today, in terms of training of professionals in this area, presents a well-diversified framework with guidelines that favor actions for individuals who need care in primary care that includes health of individuals. Consideration is given to the identification of methods used to assess incidents in primary health care, the types, contributing factors, and solutions for making early health care safer. Having as main objective to bring information about primary care and to know the importance of this debate for the professional formation of the health agent.

Method: Methodologically the study was conducted from the bibliographic research, referenced systemic descriptive, where the authors debate on the subject.

Results: The results are out of theories in this emergency field, which is primary health and the responsibility of the professional, with relevant critiques and reflections on the subject.

Conclusions: It was possible to analyze the importance, effectiveness and responsibility that the Health Agent has in primary care, becoming indispensable its role as a transformative agent regarding the health and well-being of individuals.

Keywords: Public health; Diseases; Primary attention; Health agent. 